\title{
PRESENT STATUS AND BEAM-STABILITY ISSUES OF THE KEKB INJECTOR LINAC
}

\author{
T. Suwada* , N. Akasaka, A. Enomoto, Y. Ogawa, J. Flanagan, H. Fukuma, Y. Funakoshi, \\ K. Furukawa, T. Ieiri, N. Iida, T. Kamitani, M. Kikuchi, T. Matsumoto, T. Nakamura, Y. Ogawa, \\ Y. Ohnishi, S. Ohsawa, K. Satoh, M. Suetake, H. Koiso, and K. Oide, KEK, Tsukuba, Japan
}

\section{Abstract}

The KEKB injector linac was completely upgraded for the KEK B-Factory (KEKB) project in March, 1998. Many difficulties have been overcome during the elaborate commissioning of the upgraded linac since the end of 1997. The 3.5-GeV positron and $8-\mathrm{GeV}$ electron beams have been injected to the KEKB rings with good performance. Much effort has also been continuing to stabilize the intensity and quality of the beams. Some experimental results on the beam stability issues are shown together with the recent operation status in this report. A beam test on a new scheme of a two-bunch injection was started in order to increase the positron intensity since March, 2001.

\section{INTRODUCTION}

The KEK B-Factory (KEKB) project[1] started in 1994 to test $\mathrm{CP}$ violation in the decay of $\mathrm{B}$ mesons. The KEKB comprises an asymmetric electron-positron collider with 3.5-GeV positron (LER) and 8-GeV electron (HER) rings. In such factory machines, it is strongly required that the beam stability of the injector linac be kept in order to achieve a short injection time for an effective accumulation of the integrated luminosity. The commissioning of the injector linac has continued since the end of 1997 after the original $2.5-\mathrm{GeV}$ electron linac was upgraded in order to accomplish efficient full-energy injection[2-4], even while continuing injection into two photon factory rings (PF and AR).

Many practical difficulties concerning the beamstability issues have been overcome in order to keep design beam parameters and, particularly, the good stability of the high-current primary electron beam for positron production. This effort is still being continued for further improvements.

A commissioning on two-bunch injection for the positron beam was started in order to reduce the injection time for higher storage of the beam currents, since a further increase of the single-bunch charge is difficult for a high-intensity primary electron beam from a wake-field instability point of view. Such an injection scheme is expected to halve the positron injection time, and to push ahead the KEKB experiment for an efficient accumulation of the integrated luminosity. A preliminary beam test was successfully made on two-bunch acceleration after the upgrades for the electron gun, beam-monitoring system, control software, and bunch-selection system.

\section{OPERATION STATUS}

\subsection{Four Beam Modes}

The injector linac has been operated in four beam modes. Two are injection modes for the electron and positron beams for the KEKB rings, and the others are electron injection for the PF and AR rings. The layout of the linac beam lines is shown in Fig.1 and the four beam modes are summarized in Table 1.

Table 1: Four beam modes of the injector linac.

\begin{tabular}{lcccc} 
& $\begin{array}{c}\text { KEKB/ } \\
\text { HER }\end{array}$ & $\begin{array}{c}\text { KEKB/ } \\
\text { LER }\end{array}$ & PF & AR \\
\hline Particle & $e^{-}$ & $e^{+} / e^{-}$ & $e^{-}$ & $e^{-}$ \\
Energy [GeV] & 8 & $3.5 / 3.7$ & 2.5 & 2.5 \\
Charge [nC] & 1.0 & $0.6 / 10$ & 0.2 & 0.2 \\
Repetition & 50 & 50 & 25 & 25 \\
Inj. Time [min] & $1-2$ & $4-5$ & $3-5$ & $3-5$ \\
Interval [hour] & $1-2$ & $1-2$ & 24 & 2 \\
\hline
\end{tabular}

An electron beam with a repetition rate of $50 \mathrm{~Hz}$ from the A-Gun is bunched into a single-bunch with a bunch width of 10 ps (FWHM) by two sub-harmonic bunchers (SHBs) operated at frequencies of $114.24 \mathrm{MHz}$ and 571.2 $\mathrm{MHz}$, and by a prebuncher and a buncher operated at 2856 $\mathrm{MHz}$. For the KEKB, a single-bunch electron beam with a charge of $1 \mathrm{nC} /$ bunch is accelerated up to $8 \mathrm{GeV}$ by the entire linac from the A-Gun, and a single-bunch positron beam with a charge of $0.6 \mathrm{nC} /$ bunch is accelerated up to $3.5 \mathrm{GeV}$ at the linac end, while a high-intensity primary electron beam with a charge of $10 \mathrm{nC} /$ bunch is accelerated from the A-Gun up to $3.7 \mathrm{GeV}$ at a positron target. On the other hand, a multi-bunch short-pulse beam (1-ns FW, $25 \mathrm{~Hz}$ ) with a charge of $0.2 \mathrm{nC} /$ pulse is accelerated from the C-Gun up to $2.5 \mathrm{GeV}$ for injecting into the PF and AR rings.

A reliable software operation switch changes the four beam modes without any degradation of the linac beam quality[5]. The switching time was especially reduced by introducing quick initialization of the magnet and a reliable recovery scheme of device failures. Thus, the beam modes are stably switched about 32 times/day

\footnotetext{
* Corresponding author. E-mail address: tsuyoshi.suwada@kek.jp.
} 


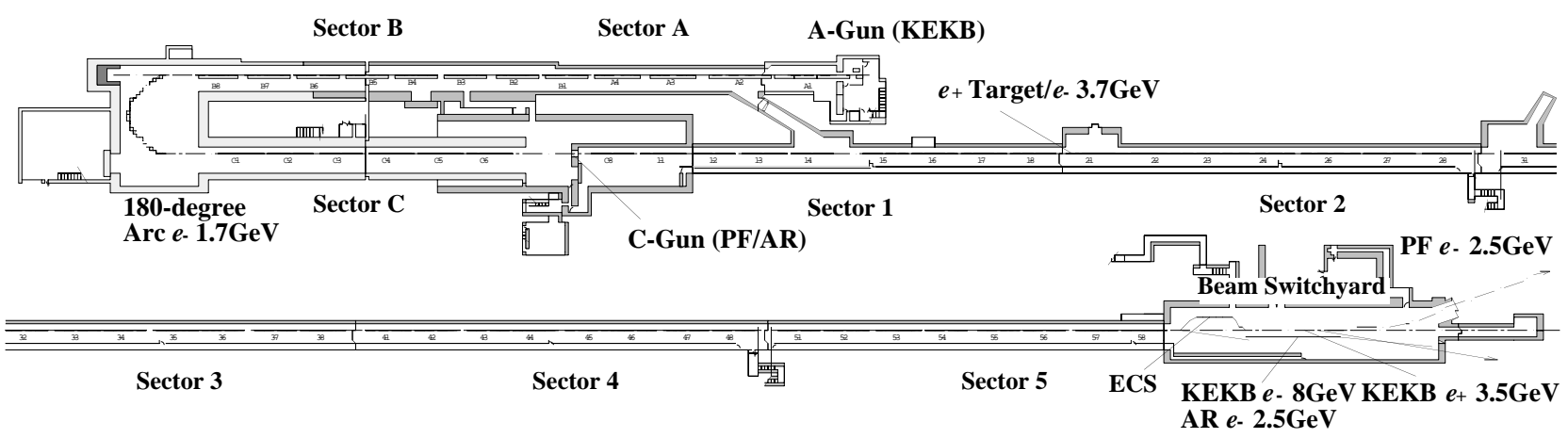

Figure 1: Layout of the KEKB injector linac.

for the KEKB injections, and one and 24 times/day for the $\mathrm{PF}$ and $\mathrm{AR}$ rings, respectively.

\subsection{KEKB Operation}

Electron and positron beams, with a charge of 0.8 and $0.4 \mathrm{nC} / \mathrm{bunch}$, respectively, are transported at the end of the beam transport line (BT). Beam injection rates of 1.8 and $4 \mathrm{~mA} / \mathrm{s}$ were recently accomplished with a repetition rate of $50 \mathrm{~Hz}$ for the LER and HER rings, respectively. Such stable high injection rates were realized by dedicated beam and rf feedback systems[5], and daily monitoring of the optics matching and beam emittances by wire scanners, and fine injection tuning. Especially the energy spread of the high-intensity primary electron beam is checked before every physics run. Typical beam parameters are summarized in Table 2.

Table 2: Typical beam parameters for the KEKB injection. The symbol "*” shows the parameters measured at the end of the linac. Subscripts 1),2) and 3) depict the parameters at the positron target, at the center of the J-arc line, and at the end of the sector $\mathrm{B}(E=1.7 \mathrm{GeV})$, respectively.

\begin{tabular}{lccc}
\hline & $e^{-} @ \mathrm{BT}$ & $e^{+} @ \mathrm{BT}$ & $e^{-} / e^{+}$ \\
\hline Energy [GeV] & 8.0 & 3.5 & $3.7^{1)}$ \\
Charge [nC] & $0.8 / 1^{*}$ & $0.4 / 0.6^{*}$ & $8.0^{1)}$ \\
$\Delta E / E[\% @ 1 \sigma]$ & 0.05 & 0.15 & $0.5^{2)}$ \\
$\gamma \varepsilon_{x} / \gamma \varepsilon_{y}[\mathrm{~mm} @ 1 \sigma]$ & $0.31 / 0.31$ & $2.4 / 2.0$ & $3.5 / 1.8^{3)}$ \\
Injection Rate & 4 & 1.8 & \\
[mA/s]@50 Hz & $(>95 \%)$ & $(>95 \%)$ & \\
\hline
\end{tabular}

\subsection{Operation Statistics}

The total operation time was 7566 hours in FY 2000, which was greatly pushed up due to full KEKB operation. The net beam-injection times for the KEKB and the photon factory rings, were 1504 and 293 hours with a loss time of less than $1 \%$. An injection availability of the linac greater than $99 \%$ for the four beam modes was accomplished.

\section{BEAM STABILITY ISSUES}

\subsection{Control of the Gun Beam}

The control and monitoring system were reported elsewhere[6] in detail. The present stability of the gun beam is briefly summarized here.

The electron gun is operated at a high voltage of $200 \mathrm{kV}$ with a repetition rate of $50 \mathrm{~Hz}$. It was found that under the typical operation condition the temperature rise is about $10^{\circ} \mathrm{C}$ at the load resistance until the gun becomes stable, which corresponds to a high-voltage variation of $1.5 \%$. A slow software feedback system was adapted in order to keep the high voltage constant. The variation of the high voltage is within $0.05 \%$ by this feedback, corresponding to a gun-beam timing of less than $1 \mathrm{ps,}$ which is measured at a wall-current monitor installed after the gun. It is also important to keep the grid-pulse timing constant, because the variation of this timing causes a different bunch shape of the beam at a different rf phase. A software feedback system is stably operated in order to keep the grid-pulse timing within 20 ps by synchronizing the timing with a reference of wave of $571.2 \mathrm{MHz}$. The phase and amplitude for the two SHBs are always monitored using the pickup rf signals from the cavities relative to the $2856-\mathrm{MHz}$ accelerating of wave by a fast sampling oscilloscope. The results show that the variations of the phase are less than 0.5 and 1 degree for SHB1 and SHB2, respectively, and the variation of the amplitude is about $1 \%$ for both the SHBs at a time scale of one week, which satisfy the stable beam transmission rate above $90 \%$ at the end of the buncher.

\subsection{Beam Trajectory Jitter Analysis}

The beam trajectory jitter quantitatively represents the beam stability concerning the transverse wake-field effect and unexpected failures for hardware devices. An application software [7] recording the beam trajectory jitter was made using data from all beam-position monitors (BPMs). The stability of the positron beam was analized based on a beam trajectory jitter measurement. The trajectory jitter measured at the BPM location is distributed on a phase space, which area can be defined as 
a jitter emittance. Figure 2 shows a variation of the horizontal jitter emittance along the whole linac beam line, where the phase space is normalized by the beam energy. The result shows that the jitter emittance of the high-intensity primary electron beam grows monotonously, except for that measured at the J-arc where the energy jitter is added, to the positron target without any step change, and for a positron beam the growth of the jitter emittance is much smaller than that of the primary electron beam. This indicates that a more careful control of the transverse wake-field effect is required for the primary electron beam. A new scheme which continuously corrects the beam orbit is under way.
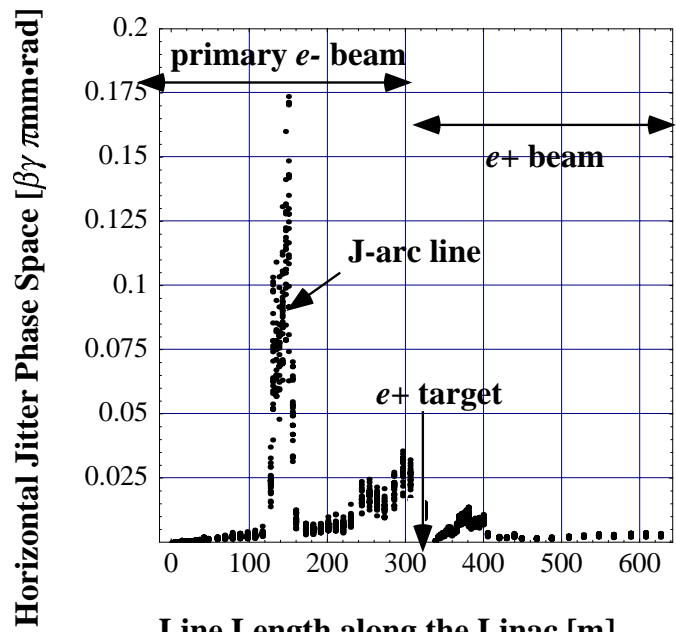

Line Length along the Linac [m]

Fig. 2: Variation of the horizontal phase space based on a positron beam trajectory jitter measurement.

\section{TWO-BUNCH ACCELERATION}

\subsection{Generation and Acceleration Schemes}

Generation and acceleration schemes of two bunches are briefly described, since they were reported elsewhere[8,9] (see also [10]) in detail. Two successive electron bunches are generated at the gun by firing two successive grid pulses with an interval time of $96.29 \mathrm{~ns}$, which are made by combining from two fast grid pulsers. The bunch interval corresponds to the period given by a common frequency of the linac and the KEKB rings. The frequency relation is summarized in Table 3. A beam-loading compensation scheme for correcting the beam energy of the second bunch was established by adjusting the timing based on the energy gain curve.

Table 3: Frequency relation between the linac and rings.

\begin{tabular}{lrcc} 
& $\begin{array}{r}\text { Multiple } \\
\text { Number }\end{array}$ & $\begin{array}{c}\text { Frequency } \\
{[\mathrm{MHz}]}\end{array}$ & $\begin{array}{c}\text { Period } \\
{[\mathrm{ns}]}\end{array}$ \\
\hline Common Frequency & 1 & 10.385454 & 96.289 \\
SHB1 & 11 & 114.240 & 8.754 \\
SHB2 & $5 \times 11$ & 571.200 & 1.751 \\
Linac & $5 \times 5 \times 1$ & 2856.000 & 0.350 \\
Ring & $7 \times 7$ & 508.887 & 1.965 \\
\hline
\end{tabular}

\subsection{Injection Test}

Bunch shapes for the two bunches were measured by an optical transition monitor with a steak-camera system after fine tuning of the grid-pulser timing of the second bunch relative to the first bunch. The result shows that each beam was single-bunched without any observational satellite bunches. The bunch length was $13-15 \mathrm{ps}$ in FWHM for the two bunches. A new orbit correction method was applied to the beam, which accomplishes the average minimum of the two-bunch orbits. After applying this method for a high-intensity primary electron beam, the two bunches were successfully accelerated up to the positron target with each bunch charge greater than $7 \mathrm{nC}$. The charge of the two positron bunches was $0.4-0.5 \mathrm{nC}$ at the end of linac. A preliminarily injection test was successfully performed using a special fill pattern, and it confirmed a bunch interval of 49 buckets in the LER. We have pushed ahead this study in order to further investigate not only the long-term stability for two-bunch acceleration, but also more realistic fill patterns for higher luminocities.

\section{CONCLUSIONS}

The KEKB injetcor linac was enthusiastically commissioned while it went into routine operation in December, 1998. The monitoring and control system of the pre-injector were reinforced in order to stabilize the high-intensity primary electron beam. The average intensity of the positron beam was remarkably increased and stable reproducibility was achieved. In order to further increase the positron intensity, a new two-bunch acceleration scheme is under testing, and a further study for long-term stability of the scheme is under way.

\section{REFERENCES}

[1] KEKB B-Factory Design Report: KEK Report 95-7 (1995).

[2] Design Report on PF Injector Linac Upgrade for KEKB: KEK Report 95-18, 1996.

[3] K.Furukawa, et al., Proc. LINAC2000, Monterey, U.S.A., 2000, p.630.

[4] A.Enomoto, et al., Proc. EPAC2000, Vienna, Austria, 2000, p.993.

[5] K.Furukawa, et al., Proc. LINAC2000, Monterey, U.S.A., 2000, p.633.

[6] S.Ohsawa, et al., Proc. EPAC2000, Vienna, Austria, 2000, p.845.

[7] T. Suwada, Proc. the 25th Linear Accelerator Meeting in Japan, Himeji, Japan, 2000, p.386.

[8] S.Ohsawa, et al., Proc. HEAC2000, Tsukuba, Japan, 2000, to be published.

[9] Y.Ogawa, et al., Proc. HEAC2000, Tsukuba, Japan, 2000 , to be published.

[10] S.Ohsawa, et al., in this conference. 